Ann. Zootech., I977, 26 (4), 585-593.

\title{
Evacuation gastrique comparée de régimes à base de fécule de pomme de terre crue ou extrudée chez le porc en croissance $\left.{ }^{(}{ }^{1}\right)$
}

\author{
L. P. BORGIDA et, $\left({ }^{*}\right)$ J. P. LAPLACE \\ avec la collaboration technique de C. Germarn \\ Labovatoive de Technologie des Aliments des Animaux \\ * Labovatoire de Physiologie de la Nutrition \\ Centre national de Recherches zootechniques, I.N.R.A., \\ $7835^{\circ}$ Jouy en Josas (France)
}

\section{Résumé}

L'étude comparative de l'évacuation gastrique (volumes et matière sèche) de deux aliments, incorporant respectivement une fécule de pomme de terre crue ou la mêtme fécule préalablement soumise à un traitement thermique par extrusion, a été réalisée chez 3 porcs. Globalement l'évacuation de la matière sèche est, en $7 \mathrm{~h}$, supé:ieure de $40 \mathrm{p}$. cent pour le régime à base de fécule extrudée (700 g contre 500). Son évacuation fait l'objet d'une régulation rapide avec alternance d'émissions importantes ou réduites de matière sèche sans variation notable du volume d'effluent. L'évacuation du régime à base de fécule crue, massive au cours de la première heure, fait ultérieurement l'objet d'une inhibition soutenue qui peut être reliée au phénomène de réplétion de l'intestin grêle proximal. Ces diverses caractéristiques peuvent être expliquées par l'hydrolyse plus ou moins facile et rapide des deux types de fécule, selon qu'il y a eu ou non traitement thermique préalable. Elles seraient susceptibles de rendre compte des modifications du bilan nutritionnel lors d'ingestion de pomme de terre crue.

\section{Introduction}

La vitesse d'hydrolyse de l'amidon par une amylase varie selon l'origine botanique et l'état physique des grains d'amidon, et selon les traitements te chnologiques appliqués au produit amylacé (AUMAITRE, I966; GrEENWOOD, I967; MERCIER, I968). On sait aussi que la vitesse d'hydrolyse in vitro de l'amidon constituant l'essentiel d'un aliment varie dans le même sens que la digestibilité de la ration, ce qui autorise l'appréciation de la valeur alimentaire d'une source amylacée à partir d'un simple test in vitro (Aumaitre, CoRring, I E DIvidich. I969). Dans ces conditions, l'amidon de pomme de terre à l'état cru n'est guère

(I) Requests for reprints to J. P. I,APLACE. 
digestible (Io à I5 p. cent) mais une simple cuisson partielle suffit à procurer une digestibilité de l'ordre de $60 \mathrm{p}$. cent (HELIENDOORN, VANDEN TOP, VAN DER WEIDE, I970). Celle-ci atteint et dépasse 90 p. cent lors de cuisson complète qui rend effectivement la pomme de terre utilisable in vivo, dans l'alimentation du porc en croissance par exemple (HENRY et RERAT, I966).

De fait, la très mauvaise utilisation de la pomme de terre crue est connue depuis longtemps, et sa piètre efficacité nutritive attribuée soit à la digestibilité médiocre de la fécule crue, rappelée ci-dessus, soit encore à une utilisation digestive apparente défectueuse de l'azote de la ration (ZELTER, CharLET-LERY, DELORTLAVAL, I 966; DELORT-LAVAL et al., I 968). Cette dernière conséquence de l'ingestion d'amidon cru de pomme de terre est considérée comme essentielle par WHITTEMORE, TAYLOR et El,SLEy (I973) dont les résultats de digestibilité de l'énergie sont, il est vrai, étonnamment élevés. Pour ces auteurs, il y aurait interférence spécifique, dans la digestion de l'azote et son utilisation ultérieure (moindre rétention) de substances solubles thermolabiles qui pourraient être des alcaloïdes.

Cette hypothèse de l'existence de substances thermolabiles inhibitrices exerçant une action au niveau métabolique a déjà été proposée par Firens (I943). Mais avant de mettre en cause de telles influences de type pharmacologique, qui pourraient au demeurant s'exercer tout aussi bien à l'égard du muscle lisse gastrointestinal, et partant du transit digestif, il paraît utile de vérifier que l'amidon, et lui seul, ne pourrait être indirectement le responsable des phénomènes observés. C'est dans cette perspective que nous avons procédé à une étude indicative de l'évacuation gastrique de régimes à base de fécule crue ou extrudée.

\section{Matériel et méthodes}

\section{I. - Protocole expérimental}

Trois pores de race Large White, pesant environ $55 \mathrm{~kg}$ ont été équipés d'une fistule duodénale réentrante selon la technique décrite antérieurement (L,APLACE, Tomassone, I970). Ces porcs habitués avant l'opération à un régime purifié riche en amidon de maïs, reçoivent dès la réalimentation post-opératoire un régime analogue dans lequel une fécule de pomme de terre crue ou extrudée a été substituée à l'amidon de maïs à raison de $5^{\circ}$ p. cent de la ration. La composition centésimale des trois aliments (préexpérimental et expérimentaux) est précisée dans le tableau $\mathrm{I}$.

Après environ 8 jours d'accoutumance à l'un ou l'autre des aliments expérimentaux, les animaux sont soumis à la collecte des effluents gastro-duodénaux avec échantillonnage puis restitution. Ces opérations ont été effectuées à l'aide du dispositif semi-automatique récemment décrit (BORGIDA, LAPI,ACE, I977). Les volumes de digesta émis et leur teneur en matière sèche, mesurée sur les échantillons prélevés (aliquote $5 \mathrm{p}$. cent), ont été enregistrés pour des périodes de 8 heures consécutives à l'ingestion d'un repas d'épreuve à $9 \mathrm{~h}$.

\section{2. - Caractérisation des aliments}

La fécule de pomme de terre crue ( 5 p. cent d'eau) a été fractionnée en 2 lots. L'un a été incorporé en l'état dans l'aliment. L'autre a subi une cuisson préalable par passage dans un extrudeur monovis industriel muni d'un manchon chauffant 
TABLEAU I

Composition des aliments utilisés (p. cent)

Feed composition (p. cent)

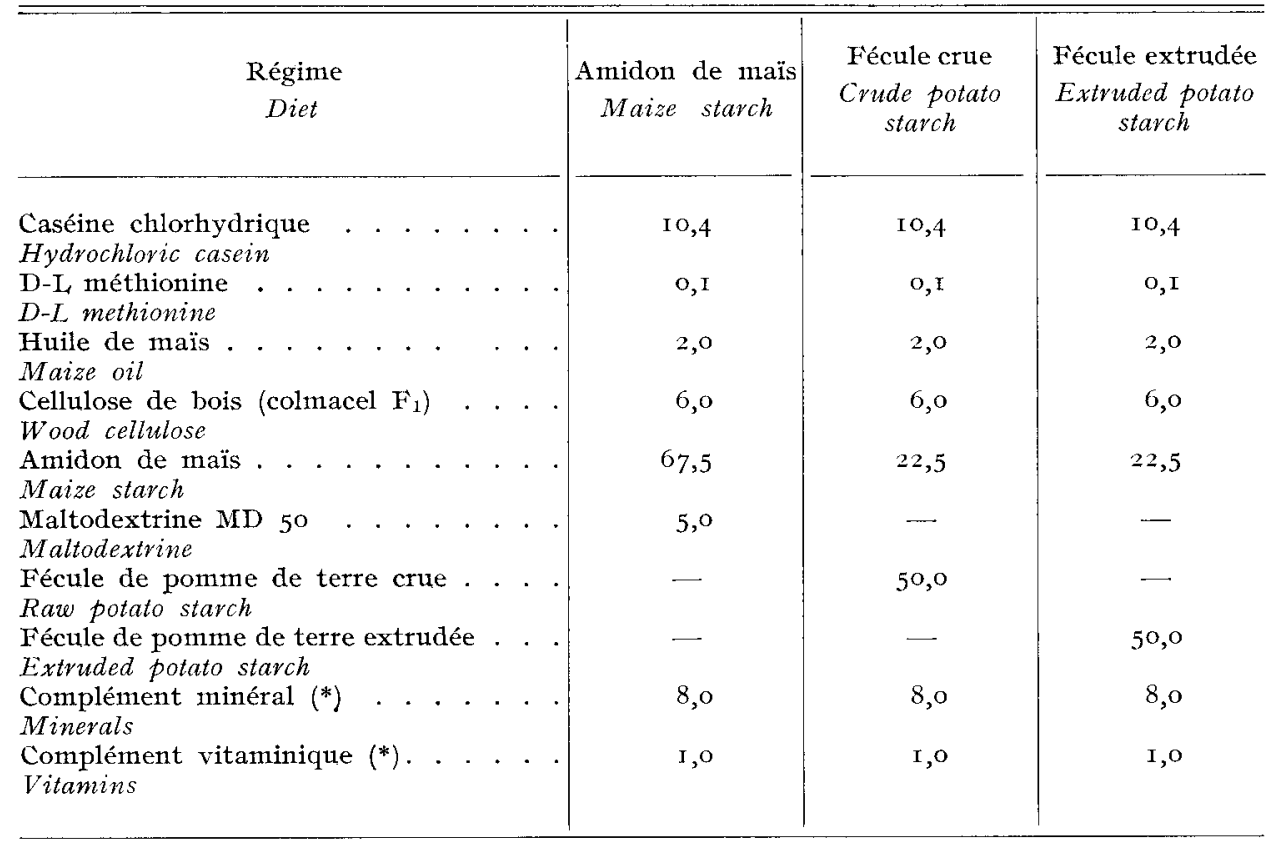

(*) D'après (from) Delort-I aval, Charlet-Iery et Zelter (ig63).

par induction, réglé pour une température d'extrusion d'environ $190{ }^{\circ} \mathrm{C}$. Cette fécule extrudée est soluble dans l'eau à $20^{\circ} \mathrm{C}$ à raison de $7 \mathrm{I}, 7 \mathrm{p}$. cent de son poids, et dans l'éthanol bouillant à raison de $0,5 \mathrm{p}$. cent seulement. La teneur en eau du produit est de 5,3 à l'extrusion et atteint I I,5 à l'équilibre.

Le régime à base de fécule crue est présenté à l'animal, après dilution par 2,5 fois son poids d'eau, comme pour le régime pré-expérimental. Le régime à base de fécule extrudée, produisant une soupe de viscosité élevée, a été dilué par 3 fois son poids d'eau pour présentation à l'animal. L'acceptabilité de ce dernier régime reste cependant faible, certains porcs en refusant systématiquement l'ingestion.

\section{Résultats}

\section{I. - Volume des effuents gastriques}

Les volumes de digesta évacués au cours des 8 heures postprandiales sont rapportés, cumulés au cours du temps, dans la figure I. Ils représentent en moyenne en $7 \mathrm{~h} \mathrm{I54,4} \pm \mathrm{I} 2,5 \mathrm{p}$. cent du volume ingéré dans le cas du régime à base de fécule 


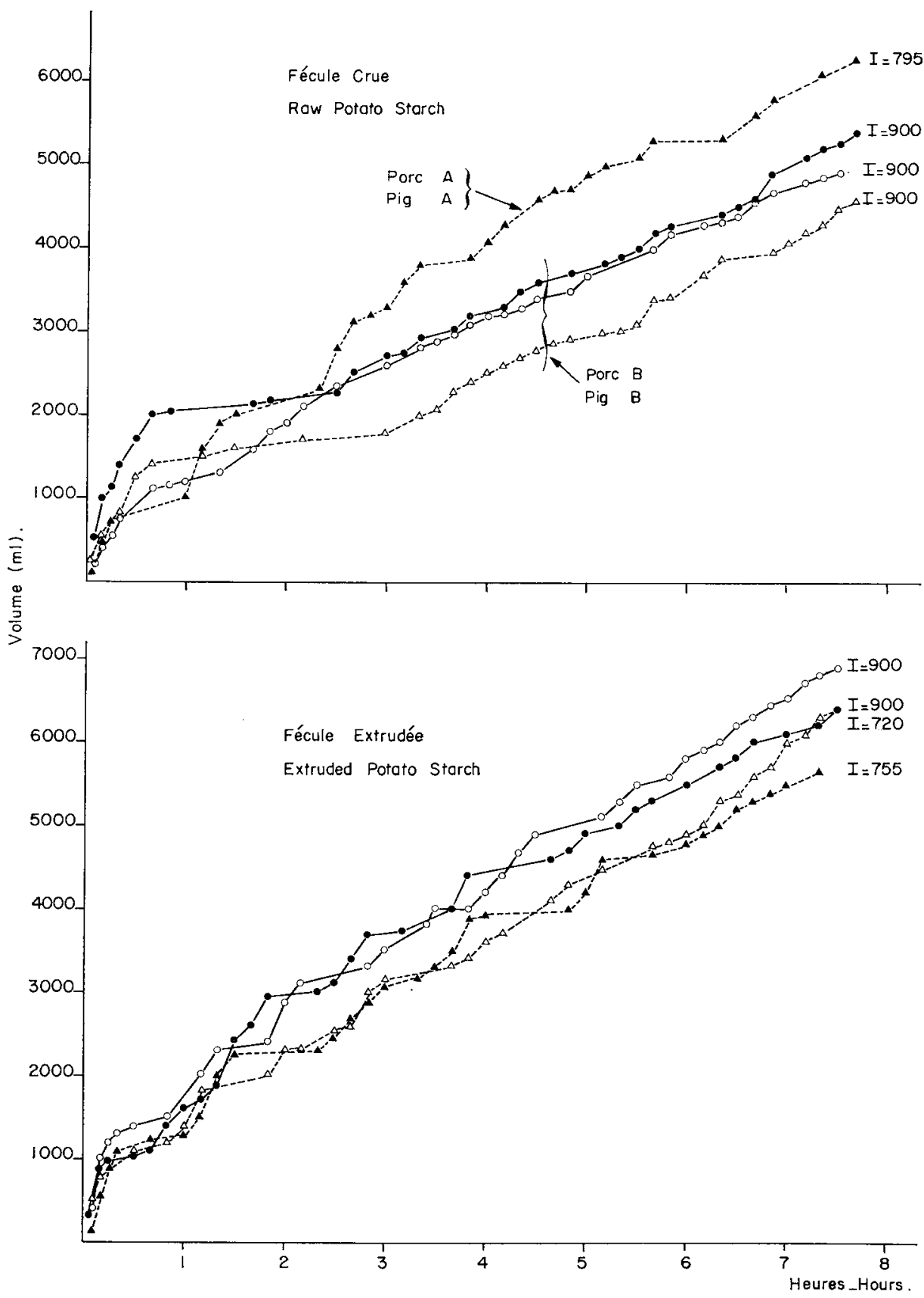

FIG. I. - Evacuation gastro-duodénale comparée de repas à base de fécule de pomme de terre crue on extrudée chez le Porc: Volumes cumulés émis au niveau d'une canule duodénale proximale chez 3 animaux de $55 \mathrm{~kg}$ environ.

$\mathbf{I}=$ quantité de matière sèche ingérée $(\mathrm{g})$.

En haut : chez 2 porcs dont l'un a subi une restriction alimentaire de 50 p. cent la veille du jour de l'épreuve (Porc A).

En bas: 4 essais chez un mêtme troisième porc.

Comparative gastro-duodenal emptying of diets with raw or extruded potato starch: Cumulated volumes collected at a proximal duodenal cannula in 3 animals $(55 \mathrm{~kg}-$ mean live weight). $\mathrm{I}=$ dry matter intake $(\mathrm{g})$.

On the top: In 2 pigs whose pig A had restricted feeding (50 per cent) during the day before test meal.

On the bottom: four test meals in the same $3^{\text {rd }}$ pig. 


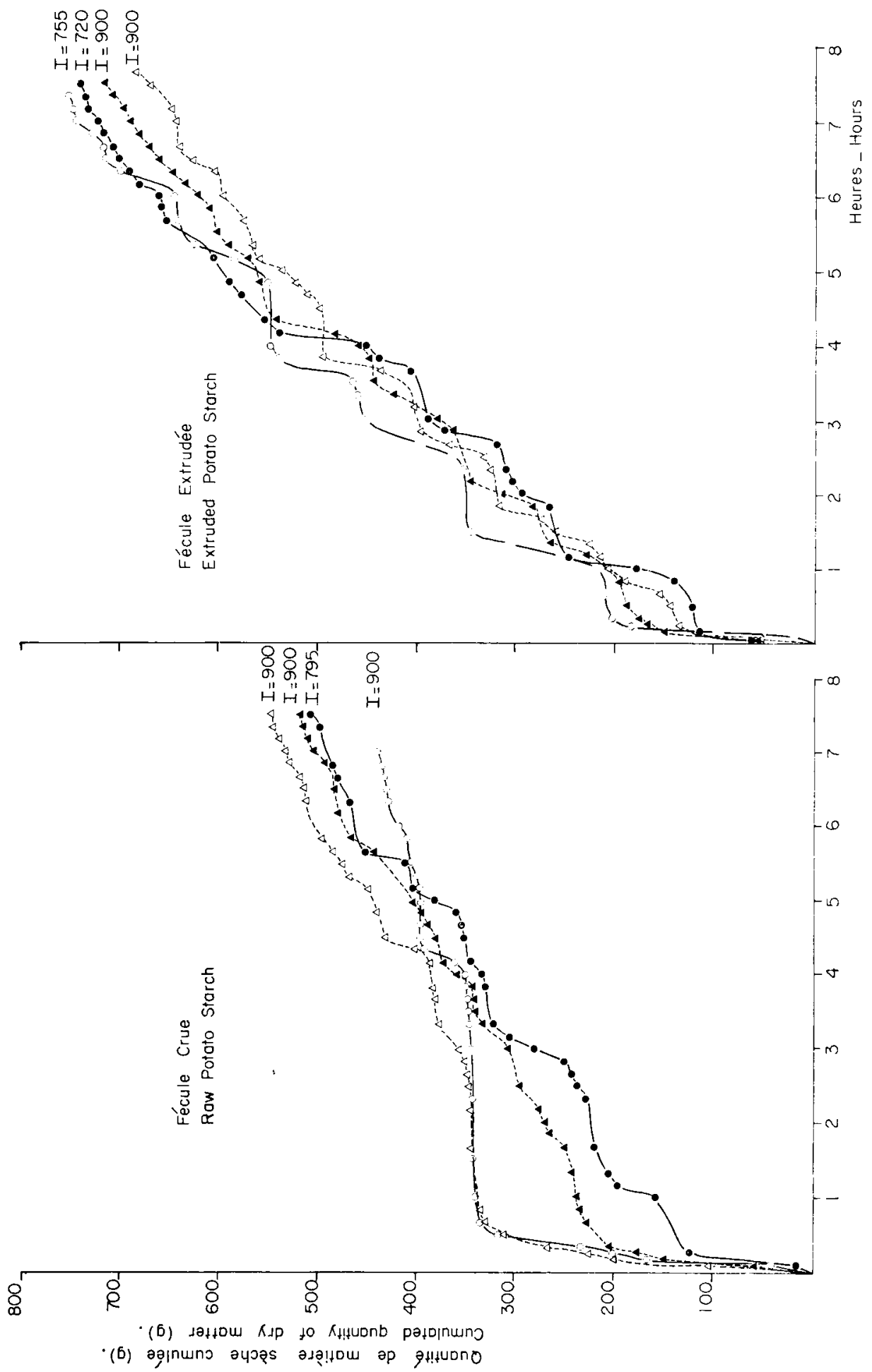

FIG. 2. - Évacuation gastro-duodénale comparée de la matiève sèche au cours des 8 h consécutives à l'ingestion de repas à base de fécule de pomme de terre crue ou extrudée, chez le Porc: donnécs recueillies lors des mêmes vepas d'épreuve chez les 3 mêmes animaux que dans la figure $I$. $\mathbf{I}=$ quantité de matière sèche ingérée $(\mathrm{g})$.

Comparative gastro-duodenal emptying of dry matter during 8 hours after test meals including vaw or extruded potato starch in the Pig: Data collected atter the same test meals in the same threc pigs as in figure $I$.

$\mathbf{I}=$ dry matter intake $(\mathrm{g})$. 
crue, et $\mathbf{I} 67,3 \pm 7, \mathrm{I}$ p. cent dans le cas du régime à base de fécule extrudée, par rapport à un volume de $I 45,7 \pm 9,5 \mathrm{p}$. cent dans le cas du régime à base d'amidon de maïs (BORGIDA, LAPI,ACE, I977).

Ces volumes totaux, comme l'aspect des courbes représentatives du volume évacué cumulé dans le temps, ne sont pas sensiblement différents de ce qui avait été antérieurement observé pour un amidon de maïs, quel que soit l'amidon incorporé au régime (fécule crue, fécule extrudée). Il n'existe pas de corrélation entre la quantité de matière sèche ingérée et le volume de digesta collectés en $7 \mathrm{~h}$. Par contre ce même volume est corrélé $(r=0,62-p<0,0$ I) avec le volume total de la ration ingérée, compte tenu de la dilution de l'aliment avant distribution, quelle qu'en soit la composition.

Enfin, ce volume total collecté en 7 h est sensiblement accru lorsque l'arimal a subi une restriction alimentaire de $50 \mathrm{p}$. cent le jour précédent celui de l'épreuve (cas du Porc A - fig. I) : il atteint par exemple 225 p. cent du volume ingéré avec le régime fécule crue.

\section{2. - Éracuation gastrique de la matière sèche}

L'analogie des courbes d'évacuation des régimes fécule crue et fécule extrudée n'est pas retrouvée sur la base de la matière sèche (fig. 2). L'aliment à base de fécule crue fait l'objet durant la $I^{\text {re }}$ heure postprandiale d'une évacuation nettement plus rapide que $1^{\prime}$ aliment à base de fécule extrudée. Mais entre la $I^{\text {re }}$ et la $4^{\mathrm{e}}$ heure, l'évacuation est considérablement ralentie pour la fécule crue, puis elle ne se poursuit qu'à un rythme relativement lent. A l'inverse celle de la fécule extrudée se poursuit d'une façon discontinue sous la forme d'émissions périodiques de quantités progressivement décroissantes de matière sèche.

Au total en 7 h, la quantité de matière sèche évacuée par l'estomac attein $t$ environ $7^{\circ 0} \mathrm{~g}$ pour le régime fécule extrudée contre $500 \mathrm{~g}$ pour le régime fécule crue.

\section{Discussion}

Compte tenu du transit différentiel des diverses fractions constituant les digesta, il est bien évident que la seule mesure du volume des effluents gastro-duodénaux peut ne pas mettre en évidence des différences importantes du rythme des apports alimentaires au contact des surfaces d'absorption. L'illustration en est apportée ici par l'absence de différence notable des volumes évacués par 1'estomac pour des régimes comportant de la fécule crue ou de la fécule extrudée alors que la seule mesure de l'évacuation gastrique de la matière sèche montre que les 2 régimes transitent d'une façon totalement différente. Ces résultats élémentaires fournissent donc un support à l'hypothèse d'une perturbation du transit digestif lors d'ingestion de pomme de terre crue. Le déterminisme du phénomène semble toutefois pouvoir être envisagé d'une façon plus simple que par le biais d'alcaloïdes hypothétiques agissant au niveau de la fibre lisse gastro-intestinale ou au niveau métabolique (WHITTEMORE, TAYLOR, EI.SLEY, I973).

Lors d'ingestion d'un aliment incorporant de la fécule de pomme de terre crue, dont nous avons rappelé précédemment la très mauvaise digestibilité, la libération de glucides solubles ne peut être que faible. Partant, l'influence inhibi- 
trice de l'évacuation, liée à la présence dans le duodénum d'holosides résultant de la digestion de l'amidon (Hun', I960) ne s'exerce qu'avec retard. Ceci peut expliquer l'évacuation massive précoce qui serait responsable, par le biais du réflexe entéro-gastrique (réplétion de l'intestin grêle proximal) de l'inhibition soutenue observée entre la I $^{\mathrm{re}}$ et la $4^{\mathrm{e}}$ heure.

La fécule préalablement soumise au traitement thermique par extrusion, particulièrement soluble dans l'eau, est d'hydrolyse beaucoup plus facile (MERCIER, I968). Ces caractéristiques autoriseraient une libération très rapide de glucides simples. Ceci peut rendre compte du freinage beaucoup plus précoce de l'évacuation de l'aliment à base de fécule extrudée. Cependant 1'absorption, rapide également, de ces glucides conduit à l'alternance de phases d'évacuation importante puis d'inhibition relative des émissions de matière sèche. La variation des quantités de matière sèche évacuée par l'estomac n'est pas associée à une variation sensible des volumes grâce à la faculté gastrique, antérieurement observée, d'émettre des effluents riches ou pauvres en matière sèche selon la quantité de celle-ci présente dans l'intestin proximal (AUFFray, MAR'TINET, RERAT, I967; LAPLACE, I975).

Par conséquent, et indépendamment des phénomènes ultérieurs de digestion bactérienne dans le gros intestin, l'apport énergétique que constitue la fécule de pomme de terre sera totalement différent dans sa quantité et dans sa cinétique selon le traitement technologique préalablement mis en ouvre. Il est probable que la modification du rythme d'évacuation gastrique ait aussi des conséquences sur la cinétique d'absorption d'autres nutriments tels que les acides aminés. Mais il faut souligner qu'indépendamment de la réduction des coefficients d'utilisation digestive apparente de l'énergie et de l'azote lors de consommation de pomme de terre crue, la simple dissociation dans le temps des apports énergétique et azoté pourrait aussi expliquer la moindre rétention azotée observée par WHITTEMORF, TAYLOR et EISLEY, (I973). On sait en effet que les glucides ne provoquent une épargne azotée que lorsqu'ils sont ingérés en même temps que les protéines, et que des acides aminés injectés par voie parentérale ne sont rapidement incorporés dans les protéines musculaires que lors d'apport entéral simultané de glucose (Munro, Black et Thomson, i959).

Les conséquences néfastes de l'incorporation dans l'aliment d'un amidon de pomme de terre crue, à l'égard du bilan nutritionnel, font l'objet d'une expérimentation complémentaire pour en évaluer l'exacte portée dans le cas de nos aliments.

S'agissant de régimes à base de fécule, c'est-à-dire d'un amidon purifié non susceptible de véhiculer un quelconque alcaloïde, les perturbations qui pourront être observées apparaîtront comme la conséquence vraisemblable du retard de l'évacuation gastrique et de la dissociation des apports énergétique et azoté, dont témoignent les résultats qui précèdent. Inversement, la recherche d'alcalö̈des présents dans le tubercule de pomme de terre cru, et éventuellement responsables des troubles décrits par WHITTEMORE, TAYLOR et EISLLEY (r973), serait justifiée si aucune perturbation n'était décelable après ingestion de fécule de pomme de terre crue.

Accepté pour publication en mai r977,

\section{Remerciements}

A la Société Roguetres National Chimie, Lille, pour la fourniture de produits (LAB $263-$ P 250) bien référencés; à Mme Mercier-GreEnwood et M. MELCion pour la caractérisation du procédé et du produit. 


\section{Summary \\ A comparative study of the gastric emptying of diets containing either raw or extruded potato starch in growing pigs}

A comparative study concerning gastric emptying (volumes and dry matter) of two diets, including either raw potato starch or the same starch previously subjected to a heat-treatment by extrusion, was made in 3 pigs. The dry matter emptying within 7 h was generally $4^{\circ} \mathrm{p}$. cent larger for the diet containing extruded starch (700 g vs $500 \mathrm{~g}$ ). A rapid regulation of the dry matter emptying with alternation of large or small emissions and without any marked variation in the volume, was observed. During the first hour, the emptying of the diet based on raw potato starch was very large whereafter a marked inhibition was noticed which could be related with the repletion of the proximal small intestine. These various characteristics might be explained by the more or less easy and fast hydrolysis of the two types of potato starches according as they had been previously subjected to the heat treatment or not. They might account for the changes in the nut itional balance after ingestion of raw potatoes.

\section{Références bibliographiques}

AUFIRAy P., MARTINET J., RERAT A., 1967. Quelques aspects du transit gastro-intestinal chez le Porc. Ann. Biol. anim. Bioch. Biophys., 7, 26I-279.

Aumaitre A., 1966. Étude de la dégradation enzymatique de divers amidons par le suc pancréatique du porcelet en cours de sevrage. VII ${ }^{\mathrm{e}}$ Congr. Int. Nutrition, Hamburg Proc., 5, IOO-IO3.

Aumaitre A., Corring T., LE Dividich J., ig69. Etude de la vitesse d'hydrolyse in vitro de quelques amidons de plantes tropicales (patate douce, banane, igname) par le suc pancréatique de porcelet. Relation entre la vitesse de dégradation in vitro et la digestibilité apparente de la ration. Journées Rech. porcine en France, Paris, 99-I 03, I.N.R.A.-I.T.P. ed.

Borgida L. P., LAplace J. P., I977. Collecte totale des contenus intestinaux chez le Porc : Automatisation de l'échantillonnage et de la restitution à l'animal, pour une étude cinétique de la digestion. Ann. Zootech, 26, 395-4II.

Delort-Laval, J., Charlet-LERy G., Zelter S. Z., r963. Efficacité de quelques protides alimentaires chez le Porc. 4) Données complémentaires sur l'action de la chlortétracycline sur le métabolisme azoté. Ann. Biol. anim. Bioch. Biophys., 3, 369-380.

Delor'-Laval, J., Cimarlet-Lery C., Zeiter S. Z., Mercier Ch,, Guilbot A., ig68. Effect of raw potato statch on nitrogen metabolism in growing pigs. Proc. 2nd World Conf. Anim. Prod., 3, 336-337.

FrEns A. M., I943. Effets nutritionnels défavorables des pommes de terre crues chez des porcs à l'engraissement. Versl. Landbouivk. Onderz., 49, 607-636.

Greenwoon C. T., I967. The thermal degradation of starch. Adv. Carbohydr. Chem., 22, 4835 I6.

Hellendookn E. W., VAN DEN TOP M., VAN DER WEIDE J. E. M., 197o. Digestibility in vitro of dry mashed potato products. J. Sci. Fd. Agric., 21, 7 I-75.

HENRY Y., RERAT A., I966. Utilisation des pommes de terre déshydratées et fraiches dans l'alimentation du pore en croissance en comparaison avec 1'orge. Ann. Zootech., 15, 23I-25I.

HUNT J. N., I960. The site of receptors slowing gastric emptying in response to starch in test meals. J. Physiol., Londres, 154, $27^{0-276 .}$

LAPLACF J. P., I975. Le transit digestif chez les monogastriques. 2) Phénomènes moteurs et mouvements des digesta. Ann. Zootech., 24, 489-552.

LAPlace J. P., Tomassone R., I970. Évacuation gastro-duodénale chez le Porc. Fistulation chronique par voie thoracique extra-pleurale; recherche d'une technique d'analyse mathématique de 1'évacuation. Ann. Zootech., 19, 303-332.

Mercifir Ch., I968. Contribution à l'étude de la structure du grain d'amidon au moyen de méthodes physiques et enzymatiques. Th. Doct. Etat. Sci. Nat., Paris. 
MUNRo H. N., BLACK J. G., Thomson W. S. T., I959. The mode of action of dietary carbohydrate on protein metabolism. Brit. J. Nutr., 13,475-487.

WIITTEMORE C. T., TAXLOR A. G., FLSLEY F. W. H., I973. The influence of processing upon the nutritive value of the potato. Digestibility studies with pigs. J. Sci. Fd. Agric., 24, 539545 .

ZELTER S. Z, Charlet'-Lery G., DelorT-LAval, J., I966. Influence de la fécule crue et cuite de pomme de terre sur les déperses azotées métaboliques et endogènes et sur la valeur nutritionnelle d'un mélange équilibré de protéines (soja + hareng) chez le Porc en croissance. $A$ cad. Agric. France, Séance du i I Mai, 567-573. 